\title{
Shuji Ogino: a critical mass of pathological epidemiologists is needed for transdisciplinary studies
}

Received: 26 November 2018; Accepted: 29 November 2018; Published: 04 December 2018.

doi: $10.21037 /$ tgh.2018.11.08

View this article at: http://dx.doi.org/10.21037/tgh.2018.11.08

\section{Editor's note}

Molecular pathological epidemiology (MPE), which was first coined in $2010(1,2)$, is an integrative field combining epidemiology and pathology, defined as "epidemiology of molecular pathology and heterogeneity of disease". It utilizes molecular pathology to incorporate interpersonal heterogeneity of a disease process into epidemiology. While pathology and epidemiology both strive to elucidate etiology of disease, MPE aims at achieving this at molecular, individual and population levels (3).

"The goal of MPE research is to study influences of the envirome on disease processes. Since the envirome differs from person to person, a disease process must differ from person to person. MPE research can play a role to study heterogeneity of any disease," explained Prof. Shuji Ogino, the pioneer in the field of MPE. As the only faculty member holding appointments in both Pathology and Epidemiology at Harvard University, he is seeking to find more cancer risk factors and more links from risk factors to tumor characteristics, which will lead to better understanding of pathogenesis. Translational Gastroenterology and Hepatology (TGH) is pleased to invite Prof. Ogino for an interview in regard to his years of MPE research.

\section{Expert's introduction}

Shuji Ogino, $\mathrm{MD}, \mathrm{PhD}$, MS, currently serves as the Chief of MPE Program, Department of Pathology, Brigham and Women's Hospital. He is also the Professor of Pathology at Harvard Medical School, Dana-Farber Cancer Institute, and Brigham and Women's Hospital; Professor in the Department of Epidemiology at Harvard T.H. Chan School of Public Health and Associate Member of Broad Institute of MIT and Harvard (Figure 1).

Prof. Ogino is recognized for his pioneering effort of establishing the interdisciplinary and integrative field of MPE. He has received a number of awards and honors, including the Ramzi Cotran Young Investigator Award from

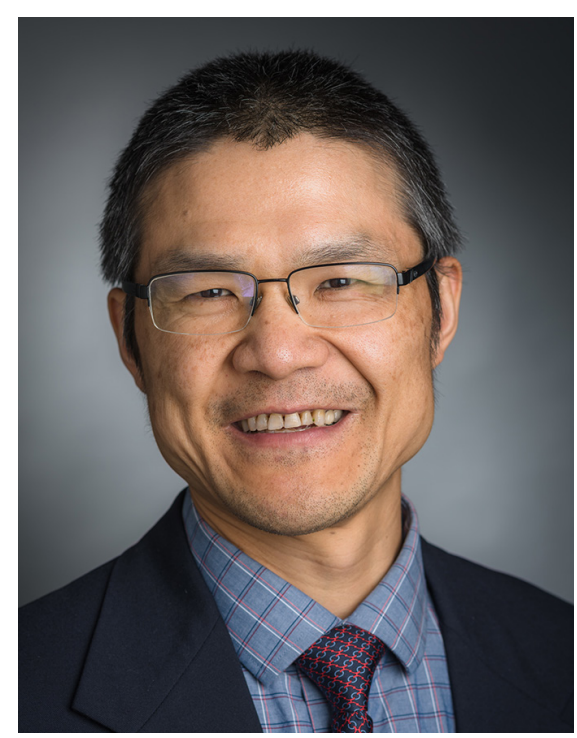

Figure 1 Prof. Shuji Ogino.

the United States and Canadian Academy of Pathology (USCAP) in 2011; Executive Officer's Award (2004) and Meritorious Service Award (2012) from the Association for Molecular Pathology (AMP); Elected Membership of American Society for Clinical Investigation (since 2014); recognition as the Most Influential Scientific Minds (2014), and High Cited Researcher (2015, 2016, 2017, and 2018) by Thomson Reuters and Clarivate Analytics; Outstanding Investigator Award from the American Society for Investigative Pathology in 2018; and a 7-year grant, National Cancer Institute (NCI) R35 Outstanding Investigator Award (2015-2022) from the National Institutes of Health (NIH).

\section{Interview}

TGH: As a pioneer in the field of MPE, what do you think are the key issues the field is critically facing right now?

Prof. Ogino: One big issue remains to be inadequate 
environment for transdisciplinary education (4). There are a number of reasons why this continues to be a big issue. Consequences of this problem is the paucity of investigators who have undergone genuinely adequate training in both pathology and epidemiology. I continue to be regarded as one unprecedented idiosyncratic pathological epidemiologist. Of course, it is good for my career in one sense, but it is not good for science. Until we have a critical mass of pathological epidemiologists, it continues to be difficult to develop transdisciplinary educational programs.

\section{TGH: You mentioned previously that "MPE encompasses all human diseases". Would you elaborate a bit more on this?}

Prof. Ogino: A certain disease, such as colon cancer, is regarded as one entity with one name. However, evidence indicates that the envirome (i.e., all factors that surround us) influences any disease process. The goal of MPE research is to study influences of the envirome on disease processes. Since the envirome differs from person to person, a disease process must differ from person to person. MPE research can play a role to study heterogeneity of any disease.

\section{TGH: What role can MPE play in the management of post-colonoscopy colorectal cancer?}

Prof. Ogino: MPE research has shown that postcolonoscopy colorectal cancer more likely has specific molecular characteristics called microsatellite instability (MSI). Cancers with high-level MSI have been known to respond to immune checkpoint inhibitors. Additional MPE research can examine whether other tumor characteristics can predict response or resistance to various therapeutic approaches including immunotherapy.

\section{TGH: How do you see the prospects of MPE? How can} MPE research discover insights into different diseases in the long run?

Prof. Ogino: Despite the big challenge mentioned above, MPE has been growing rapidly. MPE has been integrating immunology $(5,6)$. MPE research has also been expanding into a variety of diseases. In cancer research areas, it is now commonly used in studies of colorectal, breast, lung, prostate, and ovarian cancers. The MPE approach enables investigators to find more cancer risk factors and more links from risk factors to tumor characteristics, which will lead to better understanding of pathogenesis.

\section{TGH: Would you introduce us to a current NIH project that you are involved in?}

Prof. Ogino: My research program has been supported by several NIH grants. One of the major grants is the NCI R35 Outstanding Investigator Award, for which I have been the Principal Investigator (PI) since 2015. It is a 7-year multi-million-dollar grant to provide long-term stable funding for established scientists with track records as outstanding investigators. My R35 research program, entitled "Accelerating Transdisciplinary Epidemiology of Colorectal Cancer", has two major themes. The first theme is to conduct MPE research using colorectal cancer as a disease model. The second theme is to expand MPE. The expansion can be achieved through the development of analytical frameworks and statistical methods, and integrating other disciplines such as immunology and causal inference area of epidemiology into MPE. There are several other NIH grants for which I serve as a key investigator. One of them entitled "Molecular Pathological Epidemiology of Colorectal Cancer" is also a 5-year multimillion-dollar NCI U01 grant to support a large consortium of colorectal cancer studies (PI, Ulrike Peters at Fred Hutchinson Cancer Research Center). This grant attests to the pivotal role of MPE in colorectal cancer research.

\section{TGH: What are the biggest challenges encountered in the research of MPE? What have been driving you to overcome them?}

Prof. Ogino: One of the biggest challenges relates to the key issue mentioned at the first point above. Unless one has gained expertise in both fields of pathology and epidemiology, it is difficult to appreciate true power of the integrative MPE approach. The underappreciation of MPE has been a hindrance of progresses. In particular, our scientific evaluation system has been based on peer-review by scientists. You can imagine, virtually all of peer-reviewers underestimate true value and power of the MPE approach.

To address this to some extent, I established the International MPE Meeting Series in 2013, followed by four successful meetings $(7,8)$. The Fifth International MPE Meeting is being planned in June 2020 (please visit www. mpemeeting.org).

\section{TGH: What makes a good researcher?}

Prof. Ogino: In my opinion, every researcher must do 
something that no one else does. In reality, a vast majority of researchers follow others and do what others do. Therefore, if you can do unique paradigm-shifting research, it is easy to excel and become an outstanding investigator. I believe that, to be truly successful as a game-changing researcher, a researcher must conduct research in a way no one else does.

\section{Acknowledgements}

We would like to express our sincerest gratitude to Prof. Shuji Ogino for sharing his insights and opinions with us.

\section{Footnote}

Conflicts of Interest: The author has no conflicts of interest to declare.

\section{References}

1. Ogino S, Stampfer M. Lifestyle factors and microsatellite instability in colorectal cancer: The evolving field of molecular pathological epidemiology. J Natl Cancer Inst 2010;102:365-7.

2. Ogino S, Chan AT, Fuchs CS, et al. Molecular pathological epidemiology of colorectal neoplasia: an emerging transdisciplinary and interdisciplinary field. Gut

doi: $10.21037 / \operatorname{tgh} .2018 .11 .08$

Cite this article as: Li B. Shuji Ogino: a critical mass of pathological epidemiologists is needed for transdisciplinary studies. Transl Gastroenterol Hepatol 2018;3:100.
2011;60:397-411.

3. Ogino S, Lochhead P, Giovannucci E, et al. Discovery of colorectal cancer PIK3CA mutation as potential predictive biomarker: power and promise of molecular pathological epidemiology. Oncogene 2014;33:2949-55.

4. Ogino S, King EE, Beck AH, et al. Interdisciplinary education to integrate pathology and epidemiology: towards molecular and population-level health science. Am J Epidemiol 2012;176:659-67.

5. Ogino S, Nowak JA, Hamada T, et al. Integrative analysis of exogenous, endogenous, tumour, and immune factors for precision medicine. Gut 2018;67:1168-80.

6. Ogino S, Nowak JA, Hamada T, et al. Insights into Pathogenic Interactions Among Environment, Host, and Tumor at the Crossroads of Molecular Pathology and Epidemiology. Annu Rev Pathol 2018. [Epub ahead of print].

7. Ogino S, Campbell PT, Nishihara R, et al. Proceedings of The Second International Molecular Pathological Epidemiology (MPE) Meeting. Cancer Causes Control 2015;26:959-72.

8. Campbell PT, Rebbeck TR, Nishihara R, et al. Proceedings of the Third International Molecular Pathological Epidemiology (MPE) Meeting. Cancer Causes Control 2017;28:167-76.

(Science Editor: Brad Li, TGH, tgh@amegroups.com) 